\title{
Effectiveness of Intradialytic Stretching Exercises on Prevention and Reduction of Muscle Cramps among Patients undergoing Haemodialysis at PSG Hospitals Coimbatore
}

\author{
Ms.Lekha.J ${ }^{1}$, Dr.Elizabeth Jean Abraham ${ }^{2}$,Dr.G.Malarvizhi ${ }^{3}$ \\ ${ }^{1}$ Post Graduate, Department Of Medical Surgical Nursing, P.S.G College Of Nursing Peelamedu, Coimbatore- \\ 641004, Tamil Nadu, India \\ ${ }^{2}$ Principal, Subject Guide, P.S.G College Of Nursing Peelamedu, Coimbatore- 641004, Tamil Nadu, India \\ (Corresponding Author) \\ ${ }^{3}$ Vice Principal, Research Guide, P.S.G College Of Nursing Peelamedu, Coimbatore- 641004, Tamil Nadu, \\ India
}

\begin{abstract}
It is estimated that 33 to $86 \%$ of patients experience muscle cramps during haemodialysis, which results in early termination of haemodialysis session.

Objective: Evaluate the effect of intradialytic stretching exercises on muscle cramps during haemodialysis. Setting: The study was conducted at the dialysis unit-PSG, hospitals, Coimbatore.

Patients and Methods: This is a quasi experimental study. Sixty samples were selected and 30 each in both intervention and comparison group. The pre test score of muscle cramps was assessed by a standardized cramp questionnaire chart and the visual analogue scale. Intradialytic stretching exercises were given to the intervention group and the post test assessment was done on the day of $4^{\text {th }}, 7^{\text {th }}$ and $10^{\text {th }}$ day of sitting.

Results: High statistical significant improvements were noted in the pre and post interventions on muscle cramps. Comparing the intradialytic stretching exercises and the dextrose $25 \%$, stretching exercises reduces the level of muscle cramps after 6 days of therapy at $p<0.05$ level of significance.

Conclusions: The study concluded that regular stretching exercises during the haemodialysis prevent the occurrence of muscle cramps and improves the quality of life among the patients undergoing haemodialysis.
\end{abstract}

Key Words: Intradialytic, Muscle cramps, Intradialytic stretching exercises, Routine care

\section{Introduction}

Haemodialysis is a life saving measure for patients with chronic kidney disease. It is an ongoing process where patients experience complications such as hypotension, muscle cramps, disequilibrium syndrome and nausea during the procedure. Chronic kidney disease (CKD) has been increasingly recognized as a global health burden. Individuals with CKD are at risk for progressive loss of kidney function and kidney failure. One of the most common treatments for kidney failure is haemodialysis. Worldwide statistics shows that 9, 20,000 people are undergoing haemodialysis per day, which constitutes about $7-8 \%$ of the total population. C.G.Okwuonu et al (2015) mentioned that 2010 global ranking of premature causes of death show that kidney diseases moved up from position 32 in 1990 to position 24 in $2010^{1}$.Robert A Star (2012) mentioned that the beginning and ending supportive therapy for the kidney failure is haemodialysis ${ }^{2}$.

Magnard et al (2013) illustrated that haemodialysis is an epoch-making medical technology introduced clinically in the early 1960's which made it possible to prolong the lives of patients with chronic kidney disease. Haemodialysis is usually done for twice or thrice in a week with each session lasting about four or six hours. The patient quality of life can be promoted and the complications of renal failure can decrease with sufficient and effective haemodialysis ${ }^{3}$. Holley (2012) stated that muscle cramps (involuntary muscle contraction associated with severe pain) occur frequently in patients receiving dialysis. Muscle cramps can involve the legs, most commonly in the feet, but can also involve arms and hands, as well as abdominal muscles. Muscle cramps begin with fasciculations or muscle twitches and are felt to be related to nerve conduction rather than the muscles themselves.

Non- pharmacological therapy forms the cornerstone of the management of muscle cramps. It is important to discuss non-pharmacologic strategies to prevent and treat muscle cramps with patients in order to minimize pharmacotherapy. Research findings indicated that a trial of stretching program is a measure that can be done both for nocturnal leg cramps and for haemodialysis-related cramps triggered by the relaxation of the foot and ankle muscles from the prolonged recliner position for the dialysis treatment. In this regard Hallegraeff et al (2012) stated that stretching is usually a first-line treatment for cramps, and pre-bedtime stretching has been seen as an easy treatment to decrease or eliminate nocturnal cramps ${ }^{5}$. 


\section{Significance of the study:}

Muscle cramps are a common discomfort experienced by patients undergoing haemodialysis which is characterized by a sudden, painful, involuntary contraction of a muscle originates from the peripheral nerves. The most commonly affected muscles are the gastrocnemius (calf muscles), triceps (the muscles in the upper arms), the hamstrings (the muscles behind the thighs), and the quadriceps (the muscles in front of the thighs). Many non pharmacologic therapies are employed by patients prior to treatment, but there is only a little evidence to support the use of non pharmacological measures.

Almost all patients complain of muscle cramps during dialysis. They are managed with normal saline and dextrose $25 \%$ routinely in hospital. Prophylactic stretching exercises can prevent the muscle cramps. The patients can practice stretching exercises during the post dialysis period. Brenner I (2009) mentioned that regular physical activity can reduce the complications associated with CKD by inducing adaptations in the cardiovascular, nervous, and musculoskeletal systems. This increases the functional capacity and enhances quality of life in patients on HD. Haemodialysis patients can safely participate in a variety of exercise programs with minimal adverse effects. Intradialytic exercise programs that can incorporate aerobic and resistance exercise that promote exercise adherence and should be encouraged on dialysis units ${ }^{6}$.

Johansen .KL (2007) illustrated that interventions during haemodialysis sessions have become more popular and have been shown to be safe. The risks of exercise in this population have not been rigorously studied, but there have been no reports of serious injury as a result of participation in an exercise training program. It is time that we incorporate exercise into the routine care of patients who are on dialysis ${ }^{7}$.

A telephone survey was conducted in Australia and found that intradialytic exercise programs are essentially nonexistent and there is a lack of randomized, controlled trials of intradialytic exercise training. The study concluded that there is a need of intradialytic stretching exercises for haemodialysis patients, which may influence the current standard clinical practice among nephrologists and thus improve the health and quality of life of the vulnerable patients ${ }^{8}$. Hence there is a need to practice some interventions for muscle cramps during haemodialysis. Intradialytic stretching exercises help to improve the efficacy of haemodialysis thus preventing or reducing the muscle cramps.

\section{Aim of the study:}

The study aimed to evaluate the effect of intradialytic stretching exercises on muscle cramps during haemodialysis.

\section{Hypothesis:}

There will be a significant reduction in the level of muscle cramps among intervention group receiving intradialytic stretching exercises.

\section{Research Design:}

\section{Material and Methods}

Quasi experimental design pre-test post-test with control group was used to examine the effectiveness of intradialytic stretching exercises on muscle cramps during haemodialysis.

Setting: This study was conducted in dialysis unit of PSG hospitals, Peelamedu Coimbatore.

Sample: A purposive sample of 60 adult male and female patients with muscle cramps during haemodialysis. Patients were selected according to the following criteria:

1. Patients who had muscle cramps during haemodialysis.

2. Patients who are alert and cooperative.

TOOL

Section A

Part A: Demographic data: It includes age, gender, diagnosis, duration of illness, number of haemodialysis, education and occupation of the patient.

Part B: Clinical variable: It includes duration of haemodialysis treatment, sittings per week, period of experiencing cramps, location of muscle cramps, muscles involved in cramps, associated medical illness and quality of life.

Section B: Cramp questionnaire chart and visual analogue scale.

The cramp questionnaire chart was developed by Basemath.S.S.Morris

The cramp questionnaire chart was designed to assess the level of muscle cramps during hemodialysis, before and after intervention. It contains various features of muscle cramps such as the frequency of muscle cramps, duration of muscle cramps, level of pain, temperature and discomfort which was comprehensively scored as level of muscle cramps ranging from (0-13). 


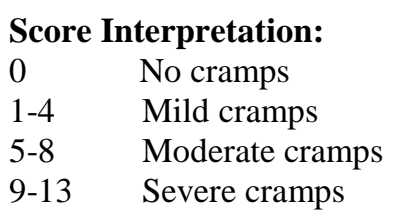

Intervention Package:

Intradialytic stretching exercises comprises of

- Ankle dorsiflexion

- Gastrocnemius stretching

- Soleus stretching

- Hamstring stretching

- Quadriceps stretching

$>$ Patients from intervention group were administered intradialytic stretching exercises during the third and fourth hour of haemodialysis.

$>$ Each session of this exercises program was for 15 minutes.

$>$ Exercises administered two times per sitting.

$>$ Exercises administered until the patients complete the post test III.

$>$ Patients from the comparison group were administered dextrose $25 \%$ during the intradialytic period.

\section{Methods}

1. Permission to carry out the study was obtained from the Medical Director and the Nephrologist after explaining the aim of the study.

2. The tool was developed on the basis of extensive review of literature, discussion with experts in the field of nursing and the investigator's professional experience. Interrater observational method was used to test the reliability of the tool. The reliability of the tool was interpreted using the 'Karl Pearson correlation' method. It has established a reliability value of ' $r$ ' 0.93

3. A pilot study conducted on $10 \%$ of the total sample who were selected based on the inclusion criteria to evaluate the content and test the feasibility, objectivity, clarity, relevancy and applicability of the study tools.

Ethical considerations: The institutional human ethics committee (IHEC), PSG institute of medical science and research had reviewed the proposal and approved the study to conduct.

\section{Techniques of data collection}

Assessment Phase:

Subjects were approached during their dialysis sessions and the purpose of the study was explained. Those who agreed to participate were given consent form to sign. Patient assessment was carried out using the tool. Pretest was done using the questionnaire and the data was collected through interview method from the patients. The muscle cramps was assessed using the cramp questionnaire chart and visual analogue scale.

The Intervention Phase:

Patients from intervention group were administered intradialytic stretching exercises during the third and fourth hour of haemodialysis. Duration of this exercises program was for 15 minutes. Exercises administered two times per sitting. Patients from the comparison group were administered dextrose $25 \%$ during the intradialytic period.

Evaluation Phase:

Evaluating the effect of the intradialytic stretching exercises was carried out during the $4^{\text {th }}$, 7 th and $10^{\text {th }}$ sitting of haemodialysis.

\section{Statistical analysis:}

Data were coded and analyzed using SPSS software package. Descriptive statistics were generated for all variables. Paired t-test was used to find the significant differences between the pre-test and post-test level of muscle cramps among patients in both the groups and Independent' $t$ ' test was used to assess the significant difference in post-test level of muscle cramps between the intervention and comparison group.

\section{Results}

Table (1) revealed that nearly half of the patients (43.3\%) belong to $45-54$ years of age from the intervention group and $(43.3 \%)$ patients from the comparison group. Majority of the patients $(50 \%)$ were male from the intervention group and (60\%) patients from the comparison group. Most of the patients $(36.6 \%)$ were diagnosed to have chronic kidney disease for more than 3 years from the intervention group and $(46.6 \%)$ patients from the comparison group. Fifty percentages of the patients were undergone 400 times of 
haemodialysis which consists of $(40 \%)$ patients from the intervention group and $(56.6 \%)$ patients from the comparison group. Half of the patients $(33.3 \%)$ were illiterate from the intervention group and (63.3\%) patients from the comparison group. Most of the patients (36.6\%) were unemployed from the intervention group and (70\%) patients from the comparison group.

Table (1): Frequency and percentage distribution of demographic profile of patients undergoing haemodialysis

\begin{tabular}{|c|c|c|c|c|c|c|c|c|}
\hline \multicolumn{9}{|c|}{$n=60$} \\
\hline Baseline data & \multicolumn{4}{|c|}{ Intervention group } & \multirow{2}{*}{\multicolumn{4}{|c|}{ Comparison group }} \\
\hline & & & & $\mathrm{n}=\mathbf{3 0}$ & & & & \\
\hline $\begin{array}{l}\text { Age and gender } \\
\text { (Age in years) }\end{array}$ & Male & $\%$ & Female & $\%$ & Male & $\%$ & Female & $\%$ \\
\hline $35-44$ & 0 & 0 & 2 & 6.6 & 4 & 13.3 & 2 & 6.6 \\
\hline $45-54$ & 5 & 16.6 & 8 & 26.6 & 6 & 20 & 7 & 23.3 \\
\hline $55-64$ & 6 & 20 & 4 & 13.3 & 4 & 13.3 & 0 & 0 \\
\hline $65-74$ & 4 & 13.3 & 1 & 3.3 & 4 & 13.3 & 3 & 10 \\
\hline \multicolumn{9}{|c|}{ Duration of chronic kidney disease (years) } \\
\hline $0-1$ & \multicolumn{2}{|c|}{11} & \multicolumn{2}{|c|}{36.6} & \multicolumn{2}{|c|}{5} & \multicolumn{2}{|c|}{16.6} \\
\hline $2-3$ & \multicolumn{2}{|c|}{8} & \multicolumn{2}{|c|}{26.6} & \multicolumn{2}{|c|}{11} & \multicolumn{2}{|c|}{36.6} \\
\hline$>3$ & \multicolumn{2}{|c|}{11} & \multicolumn{2}{|c|}{36.6} & \multicolumn{2}{|c|}{14} & \multicolumn{2}{|c|}{46.6} \\
\hline \multicolumn{9}{|c|}{ Number of haemodialysis } \\
\hline$<100$ & \multicolumn{2}{|c|}{11} & \multicolumn{2}{|c|}{36.6} & \multicolumn{2}{|c|}{3} & \multicolumn{2}{|c|}{10} \\
\hline $101-200$ & \multicolumn{2}{|c|}{2} & \multicolumn{2}{|c|}{6.6} & \multicolumn{2}{|c|}{1} & \multicolumn{2}{|c|}{3.3} \\
\hline $201-300$ & \multicolumn{2}{|c|}{4} & \multicolumn{2}{|c|}{13.3} & \multicolumn{2}{|c|}{6} & \multicolumn{2}{|c|}{20} \\
\hline $301-400$ & \multicolumn{2}{|c|}{1} & & & & & & \\
\hline$>400$ & & & & & & & & \\
\hline Education & & & & & & & & \\
\hline Illiterate & & & & & & & & \\
\hline High school & & & & & & & & \\
\hline Higher secondary & & & & & & & & \\
\hline Graduate & & & & & & & & \\
\hline Post graduate & & & & & & & & \\
\hline Occupation & & & & & & & & \\
\hline Unskilled Worker & & & & & & & & \\
\hline Professional & & & & & & & & \\
\hline House Wife & & & & & & & & \\
\hline Unemployed & & & & & & & & \\
\hline
\end{tabular}

Table (2): Frequency and percentage distribution of clinical variables of patients undergoing haemodialysis

\begin{tabular}{|c|c|c|c|c|}
\hline \multirow[t]{2}{*}{ Clinical Variables } & \multicolumn{2}{|c|}{$\begin{array}{l}\text { Intervention group } \\
\qquad \mathbf{n}=\mathbf{3 0}\end{array}$} & \multicolumn{2}{|c|}{ Comparison group } \\
\hline & $\mathbf{f}$ & $\%$ & $\mathbf{f}$ & $\%$ \\
\hline \multicolumn{5}{|c|}{ Duration of haemodialysis treatment (years) } \\
\hline $1-2$ & 0 & 0 & 0 & 0 \\
\hline $3-4$ & 9 & 30 & 3 & 10 \\
\hline$>4$ & 21 & 70 & 27 & 90 \\
\hline \multicolumn{5}{|l|}{ Hours during haemodialysis } \\
\hline 4 hours & 30 & 100 & 30 & 100 \\
\hline 6 hours & 0 & 0 & 0 & 0 \\
\hline \multicolumn{5}{|l|}{ Sittings per week } \\
\hline Twice & 18 & 60 & 13 & 43.3 \\
\hline Thrice & 12 & 40 & 17 & 56.6 \\
\hline \multicolumn{5}{|c|}{ Cramps occurred during haemodialysis } \\
\hline Middle hour & 5 & 16.6 & 15 & 50 \\
\hline Last hour & 25 & 83.3 & 15 & 50 \\
\hline \multicolumn{5}{|c|}{ Muscle cramps restrict the activities and movements } \\
\hline Yes & 30 & 100 & 30 & 100 \\
\hline \multicolumn{5}{|l|}{ Location of muscle cramps } \\
\hline Right leg & 3 & 10 & 5 & 16.6 \\
\hline Left leg & 13 & 43.3 & 12 & 40 \\
\hline Both legs & 14 & 46.6 & 13 & 43.3 \\
\hline \multicolumn{5}{|l|}{ Muscles involved in cramps } \\
\hline Calf & 15 & 50 & 21 & 70 \\
\hline Hamstring & 9 & 30 & 2 & 6.6 \\
\hline Soleus & 6 & 20 & 7 & 23.3 \\
\hline \multicolumn{5}{|l|}{ Associated medical illness } \\
\hline Diabetes mellitus & 10 & 33.3 & 10 & 33.3 \\
\hline No associated medical illness & 20 & 66.6 & 20 & 66.6 \\
\hline
\end{tabular}




\begin{tabular}{|l|c|c|c|c|}
\hline Quality of life deteriorated \\
\hline Very much & 14 & 46.6 & 10 & 33.3 \\
\hline Somewhat & 16 & 53.3 & 20 & 66.6 \\
\hline Not at all & 0 & 0 & 0 & 0 \\
\hline
\end{tabular}

As shown in table (2) ninety percentages of the patients underwent haemodialysis treatment for more than 4 years. All the patients had 4 hours of haemodialysis and experienced muscle cramps which restrict the activities of daily living. Most of the patients underwent haemodialysis twice in a week .Majority of the patients (83.3\%) were experienced muscle cramps during the last hour of haemodialysis from the intervention group and (50\%) patients from the comparison group. Nearly half of the patients $(46.6 \%)$ experienced muscle cramps in both the legs from the intervention group and (43.3\%) patients from the comparison group. Only thirty percentages of patients had associated medical illness.

Figure 1: Frequency distribution of muscles involved in cramps among intervention and comparison group

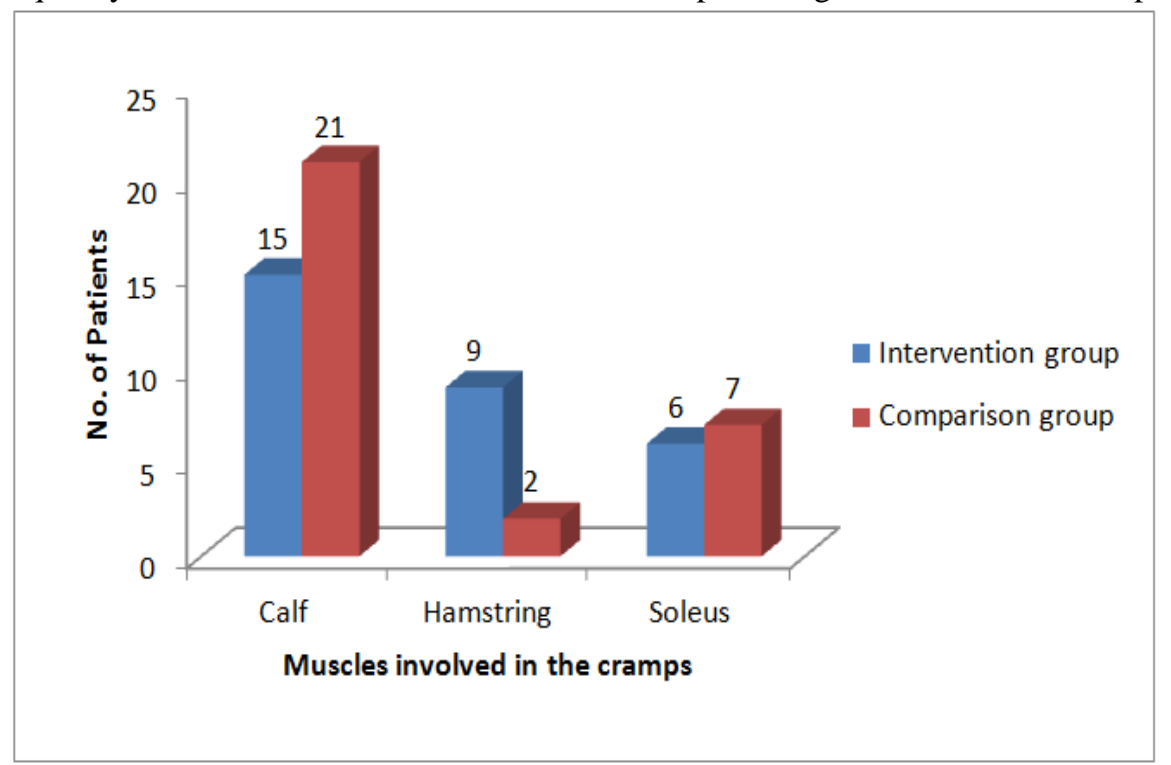

Fig. (1) It revealed that more than half of the patients experienced muscle cramps in calf muscles which include (50\%) patients from the intervention group and (70\%) patients from the comparison group.

Table (3): Comparison of intradialytic stretching exercises and routine treatment in preventing and reducing the muscle cramps between intervention and comparison group among patients undergoing haemodialysis in both post test I scores using independent ' $\mathrm{t}$ 'Test $\mathrm{n}=60$

\begin{tabular}{|c|l|r|c|c|}
\hline Sl. No & \multicolumn{1}{|c|}{ Study Group } & Level of muscle cramps & \multirow{2}{*}{ ' $\mathbf{t}$ ' Test } & \\
\cline { 3 - 3 } & & Mean \pm SD & & \\
\hline 1. & Intervention group & $5.93 \pm 4.98$ & \multirow{2}{*}{$0.146^{\mathrm{NS}}$} & \multirow{2}{*}{2.02} \\
\hline 2. & Comparison group & $6.1 \pm 5.61$ & \\
\hline
\end{tabular}

NS- Not Significant

Table (3) describes that there is no significant difference in the level of muscle cramps after 2 days of intradialytic stretching exercises.

Table (4): Comparison of intradialytic stretching exercises and routine treatment in preventing and reducing the muscle cramps between intervention and comparison group among patients undergoing haemodialysis in both post test II scores using independent' $\mathrm{t}$ ' Test $\mathrm{n}=60$

\begin{tabular}{|c|l|r|c|c|}
\hline Sl. No & Study Group & Level of muscle cramps & ' $\mathbf{t}$ ' Test & ' $\mathbf{t}$ ' table value \\
\cline { 3 - 3 } & & Mean \pm SD & \multirow{2}{*}{$0.541^{\mathrm{NS}}$} & \multirow{2}{*}{2.02} \\
\hline 1. & Intervention group & $4.26 \pm 4.80$ & \\
\hline 2. & Comparison group & $4.96 \pm 5.21$ & 0.2 \\
\hline
\end{tabular}

NS- Not Significant

Table (4) revealed that there is no significant difference in the level of muscle cramps after 4 days of intradialytic stretching exercises. 
Table (5): Comparison of intradialytic stretching exercises and routine treatment in preventing and reducing the muscle cramps between intervention and comparison group among patients undergoing hemodialysis in both post test III scores using independent ' $t$ ' Test $n=60$

\begin{tabular}{|c|l|c|c|c|}
\hline \multirow{2}{*}{ Sl. No } & Study Group & Level of muscle cramps & ' $t$ ' Test & 't' table value \\
\cline { 3 - 3 } & & Mean \pm SD & \multirow{2}{*}{$10.046^{*}$} & \multirow{2}{*}{2.02} \\
\hline 1. & Intervention group & $2.10 \pm 3.67$ & \\
\hline 2. & Comparison group & $2.63 \pm 2.85$ & & \\
\hline
\end{tabular}

Statistically Significant $-* \mathrm{p}<0.05$

Table (5) it was observed that all patients from the intervention group had reduction in the level of muscle cramps after performing the intradialytic stretching exercises. There is a significant improvement in the level of muscle cramps after 6 days of therapy.

\section{Discussion}

The present study results indicated that there is effectiveness in performing intradialytic stretching exercises on prevention and reduction of muscle cramps during haemodialysis. The current study findings revealed that among 30 patients in intervention group, majority of the patients $(36.6 \%)$ patients experienced severe muscle cramps after 2 days of intervention. More than half of the patients $(63.3 \%)$ patients did not experience cramps after 4 days of intervention. Majority of the patients (66.6\%) patients did not experience muscle cramps after 6 days of intervention. This result describes the effect of intradialytic stretching exercises in reducing the muscle cramps during haemodialysis.

These findings were in line with the results of a study performed by Magda Mohamed, Amal Mohamed, Shalabia Abo Zead (2007) they found that performing intradialytic stretching exercises reduces the level of muscle cramps were statistically significant with the level of significance at $p<0.05^{9}$. More than half of the patients experienced muscle cramps in calf muscles. Coinciding with these results Naylor, Young (2004) mentioned that $83 \%$ of the patients are experiencing muscle cramps in the calf muscles ${ }^{10}$.

The present study findings revealed that there were statistical significant difference in reducing the frequency of muscle cramps at $\mathrm{p}<0.05$ level of significance while comparing the intradialytic stretching exercises and dextrose 25\%. Thus intradialytic stretching exercises reduce the level of muscle cramps after 6 days of therapy. These results are in agreement with Hallegraeff. J.M, et al (2013) emphasized the effect of calf stretching exercises in prevention of muscle cramps during haemodialysis ${ }^{11}$. The present study results showed that there were no significant difference between the intradialytic stretching exercises and the routine treatment after 4 days of intervention. Only after 6 days of intervention there was a significant difference between the intervention at $\mathrm{p}<0.05$ level of significance. The study shows that there is effectiveness in the prevention and the reduction of muscle cramps during haemodialysis after longer days of therapy. This study concluded that continuous Intradialytic stretching exercises can prevent and reduces the level of muscle cramps during haemodialysis. Study findings corroborates with Canzanello. V.J, et al (2006) data in which continuous stretching exercises reduces the level of muscle cramps during haemodialysis ${ }^{12}$.

There was no association between the level of muscle cramps and the demographic variables. Coinciding with these results, Brass et al (2007) found that peripheral arterial disease has no relationship on muscle cramps ${ }^{13}$.

\section{Conclusions}

Muscle cramps are the most prevalent intradialytic complication and it is a subjective feeling that can be expressed by all the patients. There are many pharmacological and non pharmacological measures are adopted to treat the muscle cramps but the data from various studies revealed that there was no complete relief from the cramps. This study was undertaken to assess the effectiveness of intradialytic stretching exercises on prevention and the reduction of muscle cramps among patients undergoing haemodialysis at PSG hospitals, Coimbatore. Intradialytic stretching exercises are an effective method which can be used as a preventive therapy in the treatment of muscle cramps. By conclusion intradialytic stretching exercises and the administration of dextrose helps to reduce the level of muscle cramps. Regular stretching exercises for a week will reduce the level of muscle cramps and also prevent the occurrence of cramps during haemodialysis.

\section{Recommendations}

$>$ Training can be provided to the staff nurses regarding passive intradialytic stretching exercises.

$>$ Structured teaching programme on active intradialytic stretching exercises can be provided to the patients undergoing haemodialysis.

$>$ Encourage the patients to note the frequency of muscle cramps in a diary after performing the exercises.

$>$ Intradialytic stretching exercises can be adapted as a procedure to the patients undergoing haemodialysis.

$>$ Nurses can introduce the evidenced based practice of doing these stretching exercises during the haemodialysis session. 
$>$ Nurses must emphasize the patients comfort during haemodialysis thus helps to reduce the muscle cramps experienced by the patients.

\section{References}

[1]. Abali,C., Adejumo,O., Chukwuonve,I., Ogah,S.O., Okwuonu,C.G. (2013). Awareness level of kidney functions and diseases among adults in a Nigerian population. Journal of American Science, 25,158-163.

[2]. Robert A Star. Treatment of acute renal failure. International Journal of Kidney, 2015; 54(3): (accessed doi:10.1046/j.15231755.19980020).

[3]. Magnard et al. Effects of a six - month intradialytic physical activity program and adequate nutritional support on protein energy wasting, physical functioning and quality of life in chronic haemodialysis patients. British Medical Journal, (2013): (accessed 14.259-261.doi:10.1186/1471-2369-14-259).

[4]. Holley, et al. . Muscle cramps in dialysis patients. CANNT Journal2012; 25(3).

[5]. Hallegraff, et al. An effective stretching regimen to prevent nocturnal leg cramps. Journal of Physiotherapy 2012; 59(4): (accessed doi: 10.1061/S1836-9553).

[6]. Brenner. (2009). Exercise performance by hemodialysis patients. Journal of Basic And Clinical Renal Science 37.84-96.

[7]. Johansen KL... Exercise in the end-stage renal disease population. Journal of American Society of Nephrology. 2008; 18(6).

[8]. Cheema, BS ., Singh, MA, Smith, BC. A rationale for intradialytic exercise training as standard clinical practice in ESRD. American Journal of Kidney Diseases, 2005; 45(5): (accessed doi:10.1053/j.ajkd2005.01.030).

[9]. Amal Ahmed. Magda Mohamed., Shalabia Abo Zead. Impact of stretching exercises protocol on reduction of muscle cramping during haemdialysis, among chronic renal failure patients. Australian Medical Journal 2007; 5(3):

[10]. Naylor, J.R., Young J.B. (2004).A general population survey of rest cramps. Age Ageing. Australian Journal of Nephrology. 23.418-420

[11]. Canzanello et al. Hemodialysis associated muscle cramps. British Journal of Medicine 2006; 5(4): (accessed doi: 10.111/j1525139.19921600234.)

[12]. Brass Adler et al. Peripheral arterial disease is not associated with an increased prevalence of intradialytic cramps in patients on maintenance hemodialysis. American Journal of Nephrology 2007; 22(5-6) 